\title{
How to Use Sputtering for Achieving Nanocomposite Structures
}

\author{
Nuno Figueiredo ${ }^{1}$, Albano Cavaleiro ${ }^{1,2}$ \\ ${ }^{1}$ SEG-CEMMPRE \\ Mechanical Engineering, University of Coimbra, Coimbra, Portugal \\ ${ }^{2}$ IPN - LED\&MAT \\ Instituto Pedro Nunes, Coimbra, Portugal \\ nuno.figueiredo@dem.uc.pt; albano.cavaleiro@dem.uc.pt
}

Nanocomposite coatings containing metal nanoparticles are receiving increasing interest from both researchers and industry. One of the main properties of the metal nanoparticles that is of interest is their ability to support Localized Surface Plasmon Resonances (LSPRs), optically induced oscillations of free electrons at the surface of the metal nanoparticles. The excitation of LSPRs by incoming light results in strong light extinction effects that are heavily dependent on the nanoparticle's dielectric constant, size, shape and concentration and also on the dielectric constant of the surrounding medium. Since for each application a specific LSPR signal is needed, it becomes of paramount importance to be able to produce nanocomposites with good control over the nanoparticle's morphology and over its distribution in the matrix material.

Among the various production methods available for obtaining nanostructured coatings, the sputtering technique has become widely used due to its low cost, simplicity in the process, flexibility with the materials and remarkable coating quality. In this work, Au nanoparticles were incorporated into four different matrixes: $\mathrm{Al}_{2} \mathrm{O}_{3}, \mathrm{WO}_{3}, \mathrm{TiO}_{2}$ and $\mathrm{AlN}$. These matrixes have distinct refractive indexes, which allow varying the LSPR throughout the visible region. The LSPR-exhibiting nanocomposites were obtained using three different sputtering techniques, with the aim of obtaining nanocomposites with good control over the nanoparticle's morphology and distribution in the matrix: (i) co-sputtering followed by thermal annealing treatments up to $500^{\circ} \mathrm{C}$; (ii) alternating-sputtering using pulsed DC power sources and (iii) alternating-sputtering using a plasma gas condensation (PGC) nanoparticle source.

The first method of deposition allowed achieving nanocomposites with a relatively homogeneous dispersion of spherical Au nanoparticles within the matrixes. The Au integration in the matrix was found to be strongly dependent on the chemistry of the matrix and also on the concentration of noble metal. The application of thermal annealing treatments allowed increasing the Au nanoparticle sizes (up to $8 \mathrm{~nm}$ ) and also affected the dielectric properties of the matrix. The second method allowed a more effective and independent control over the nanoparticles morphologies without the need of any thermal annealing treatment. Very thin Au layers were deposited, intercalated with the oxides, allowing the formation of Au nanoparticles with different island-type morphologies. With the $\mathrm{Au}$ content the mean nanoparticle size increased progressively (up to $15 \mathrm{~nm}$ ) whereas the shape of the nanoparticles evolved from rather spherical to spheroidal, with decreasing aspect ratios. The decrease in the nanoparticles aspect ratios resulted in more intense and red-shifted LSPR absorption bands. The third method allowed the production of large amounts of nanoparticles with a high level of control over its mean size. Au spherical nanoparticles with sizes between 5-65 nm were deposited with varying deposition rates and size dispersions. Nanocomposites incorporating $\sim 15 \mathrm{~nm}$ Au nanoparticles were obtained with good control over the nanoparticle size and distribution, demonstrating the very high potential of using this technique for nanostructured coatings. Additional experimental work was performed with new apertures that allowed achieving a larger deposition area for the PGC source. This can be of special interest to the coating industry.

\section{References}

[1] N.M. Figueiredo, "Nanocomposite coatings based on metal nanoparticles exhibiting surface plasmon resonance deposited by sputtering techniques", PhD Thesis, University of Coimbra, August 2017. 